\title{
Stochastic analysis of impact mixing of bulk materials in a rotary apparatus
}

\author{
Anna Kapranova ${ }^{1,{ }^{*}, \text { Daria } \text { Bahaeva }^{1}, \text { Dmitry Stenko }}{ }^{1}$, Daria Fedorova $^{1}$, Alexander Vatagin ${ }^{1}$, and Anton Lebedev $^{1}$ \\ ${ }^{1}$ Yaroslavl State Technical University, Yaroslavl, Russian Federation
}

\begin{abstract}
The purpose of this work is to analyze the distribution of bulk materials according to the characteristic parameters of the mixing process at the second stage of processing in a rotary apparatus. The design of the rotary device has a wide range, including for the needs of agriculture, for example, when forming mixtures of mineral fertilizers and multifunctional pyrethroid drugs. At this mixing stage, impact interaction with the bump surface of rarefied flows of bulk components occurs, obtained by scattering the rotating drum by elastic blades at the previous stage of the technological chain. The obtained dependencies between the characteristic angle of dispersion of bulk materials by the specified mixing drum (at the first stage) and the angle of reflection of the corresponding flows from the baffle surface (at the second stage) are used in the stochastic analysis of impact mixing. Modeling of the differential functions of the distribution of the number of particles of mixed bulk components by the angle of reflection from the baffle surface is carried out taking into account the physical and mechanical characteristics of the working materials, design parameters, and operating parameters of the rotary apparatus. The calculation results made it possible to reveal the effective ranges of variation of the characteristic parameters for the shock mixing of bulk materials in a rotary apparatus.
\end{abstract}

\section{Introduction}

The processes of processing bulk materials are important elements of various technological chains in the food and chemical industries, agriculture, and the construction industry. At the same time, one of the most common technological operations is the process of mixing solid dispersed components, including a granular and powder structure. For example, this problem is of particular relevance when obtaining free-flowing mixtures for the needs of agriculture when forming mixtures of mineral fertilizers [1,2] and multifunctional pyrethroid drugs [3]. However, the use of inhomogeneous mixtures with a complex composition of mineral fertilizers or pyrethroids can cause irreparable harm not only to various varieties of cultivated plants but also affect the state of the ecosystem. For example, the quality of the soil, its microflora, harm the animal world and human health $[4,5]$. Note that the toxic properties of some mixed components for special purposes [6] impose certain restrictions on experimental studies of the conditions for their effective mixing. This circumstance dictates the need to solve verification problems in the system analysis of the process under study $[7,8]$ with the selection of working substitute materials with similar physical and mechanical properties

The purpose of this work is to analyze the distribution of bulk materials according to the characteristic parameters of the mixing process at the second stage of processing in a rotary apparatus [9]. At this stage of mixing, there is an impact interaction with the bump surface of rarefied flows of granular components obtained by scattering the elastic blades of a rotating drum at the previous stage of the technological chain [10]. Interest in the design of new devices for this purpose is explained by the specific properties of the processed working materials and the specific conditions of the technology for obtaining the finished product in the form of their high-quality mixture [11]. Difficulties in predicting the degree of homogeneity of a given mixture, corresponding to a given technological regulation, can be successfully solved already at the design stage of the mixer, which is based on a systemstructural analysis of the mixing process $[8,12,13]$.

The description of the theoretical basis of the specified forecasting is associated with the choice of the criterion for assessing the quality of the mixture of processed bulk components [14-16], which reflects the preference in the application of the stochastic approach to modeling this technological processing process $[17$, $18]$.

However, indeterminate methods for assessing the volume fraction of a key component in a ready-made free-flowing mixture have various modifications, for example, cybernetic [19, 20], time series [21-23], Markov AA chains. [24-27]. In particular, the last of the above, the Markov approach, is one of the most actively developing in recent years [27-31]. Within its framework, the energy method described in the works of Yu. L. Klimontovich $[32,33]$ and is proposed for use in

\footnotetext{
* Corresponding author: kapranova anna@,mail.ru
} 
this work. The energy method [32, 33] has been adopted by the authors to solve some problems in chemical technologies, in particular, in models of bubble-type hydrodynamic cavitation [34-36], fragmentation of liquid droplets [37]. This method has been tested in modeling the processes of formation of rarefied flows of granular and/or powder materials when they are mixed in devices with a moving belt [14], devices with gravitational action $[13,16,17]$, etc.

At the same time, the solution to some related problems is of particular interest. The priority tasks include the following. First, it is required to identify the most significant factors influencing the performance of the investigated technological operation while weakening the effects of segregation and agglomeration, the adhesive properties of working materials [29, 31]. In particular, the appropriate selection is made from the selected parameters of the design of the rotary mixer [9] and the characteristics for the modes of its operation both at the first [10] and at the second stages of this process. Secondly, it is necessary to establish the effective ranges of variation of the parameters identified by the specified criterion.

\section{Description of the relationship between the characteristic parameters of the mixing process of bulk components at its two stages}

The successful implementation of one of the promising stages in the processing of granular and/or powder materials, such as mixing these components in rarefied flows, is observed in impact-type devices. For example, the creation of these rarefied flows is ensured when the mixing elements operate in the form of radial brushes, beats with a helical winding in one [14] or two directions $[13,15-17]$, elastic blades $[9,10]$ on a rotating drum. Thus, in the projected continuous-action rotary apparatus [9], as noted earlier, there is a division of the mixing process into two appropriate stages: (1) obtaining rarefied flows of granular and/or powder components by scattering their particles with elastic blades [10]; (2) impact mixing of components when interacting with the bump surface (Fig. 1). Both stages presented are carried out on a horizontal conveyor belt 1 . With the help of this conveyor, first, the layers of mixed materials are delivered, vertically loaded into the working area of the apparatus, into the gap with the mixing drum. After interacting with its elastic blades (stage 1 [10], mixing), the particles of bulk materials $(i=1,2)$ in the created rarefied flows, hitting the inclined bumper 2 , return to the moving belt, forming a mixture (stage 2, mixing, (Fig. 1). Additionally, the resulting mixture of bulk materials is compacted by a roller without elastoplastic deformations of the grains (stage 3, deaeration) and unloaded from belt 1 .

The particles of the component $i=\overline{1, n_{i}}$ with the true density of matter $\rho_{T i}$ have the shape of a sphere with a diameter $d_{T i}$, which corresponds to the following averaging over each fraction of the particle size distribution $d_{T i}=n_{v}^{-1} \sum_{v=1}^{n_{v}} d_{T i v}$.

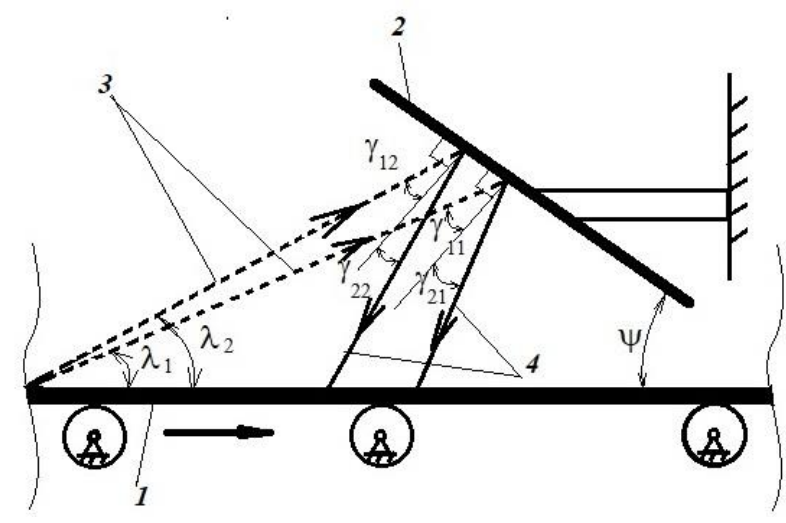

Fig. 1. Conditional scheme of impact mixing of bulk materials: 1 - conveyor belt; 2 - bump surface (inclined bumper); 3 and 4 - directions of the averaged particle velocities in rarefied flows ( ): "falling" on the baffle surface (after the completion of stage 1) and "reflected" from it (the result of stage 2).

The process of mixing bulk materials at stage 1 received a stochastic description in work [10] when setting the parameters: constructive $a_{j_{1}}=\left\{R_{b}, L_{b}, p_{0}, k_{0}, \mu, h_{r}\right\}, \quad j_{1}=\overline{1, n_{1}}, n_{1}=6 ; \quad$ regime $b_{j_{2}}=\left\{\omega, h_{0}, h_{L}\right\}, \quad j_{2}=\overline{1, n_{2}}, n_{2}=3$; physical and mechanical $\quad c_{j_{3}}=\left\{\rho_{T i}, D_{T i}, k_{u}\right\}, \quad i=\overline{1, n_{i}}, \quad j_{3}=\overline{1, n_{3}}$, $n_{3}=2 n_{i}+1$. Here it is indicated: $R_{b}$ is radius of the mixing cylindrical drum; $L_{b}$ is the length of its elastic blades of rectangular shape with attachment in the tangent plane to the surface of the drum; $p_{0}$ is number of blades; $k_{0}$ is number of their ranks; $\mu$ is offset angle when installing these rows; $h_{r}$ is row spacing; $\omega$ is drum rotational speed; $h_{0}$ is minimum distance between drum and belt surfaces; $h_{L}$ is total height of layers of bulk components on the belt; $k_{u}$ is coefficient of angular stiffness of an elastic blade. Also, the characteristic of the degree of deformation of the blades in the gap between the drum and the conveyor belt is introduced $\delta=h_{0} / L_{b}$.

For the operation of mixing bulk materials at stage 2, the following design parameters of the apparatus are additionally taken: $\psi$ is bump angle; $H_{1}$ is respectively, the characteristic distances along the conveyor belt between the drum and the bump surface. Then the updated set of design parameters takes the form: $a_{j_{4}}=\left\{a_{j_{1}}, \psi, H_{1}\right\}, j_{4}=\overline{1, n_{4}}, n_{4}=n_{1}+2$.

To obtain functional dependencies between the main characteristic parameters of the mixing process of bulk components, we introduce additional angles that describe the behavior of particles during the movement of rarefied flows in the working volume of the apparatus. Assume that $\theta_{i v}$ is the characteristic angle of scattering of material particles $i=\overline{1, n_{i}}$ blades of each row $v=\overline{1, k_{0}}$ 
(stage 1), which is measured from the vertical axis of the coordinate system associated with the center of the Archimedes spiral $r_{A_{i v}}\left(\theta_{i v}\right)=u_{1}+u_{2} \theta_{i v}$. On a given curve $r_{A_{i v}}\left(\theta_{i v}\right)$ the ends of the deformed blades are located, where it is accepted: $u_{1}=R_{0}+h_{0}$; $u_{2}=\left\{\left[R_{0}^{2}+\left(R_{b}+L_{b}\right)^{2}\right]^{1 / 2}-u_{1}-h_{0} / 3\right\} / \gamma ;$ $\gamma=\pi+\operatorname{arctg}\left\{\left(\mathrm{R}_{b}+L_{b}\right) / R_{0}\right\} ; R_{0}=\left(1+\cos \varphi_{0}\right) R_{b}$.

Moreover, we introduce additionally $\lambda_{i}=k_{0}^{-1} \sum_{v=1}^{k_{0}} \lambda_{i v}$ is the angle of dispersion of particles from the flow of a bulk component $i=\overline{1, n_{i}}$ (stage 1). Here the value $\lambda_{i v}, v=\overline{1, k_{0}}$ corresponds to the average scattering angle of particles for each row of blades with a total number when counting from the conveyor belt to the direction of the speed of movement of material particles $i=\overline{1, n_{i}}$. The movement of the particles of the components is characterized by the averaged values of the angles of "incidence" on the bump surface $\gamma_{1 i}=k_{0}^{-1} \sum_{v=1}^{k_{0}} \gamma_{1 i v}$ and "reflections" from it $\gamma_{2 i}=k_{0}^{-1} \sum_{v=1}^{k_{0}} \gamma_{2 i v}$ for the directions of the averaged particle velocities in rarefied flows directed tangentially to the Archimedes spiral $r_{A_{i v}}\left(\theta_{i v}\right)$. Note that the velocity vector of a spherical particle is determined by the velocity of its center of mass $\mathbf{v}_{C_{i v}}\left(v_{x i v}, v_{y i v}\right)$ in a Cartesian coordinate system associated with the center on the axis of rotation of the drum. The following relation was obtained, which is consistent with the data of [17], between the values of the scattering angles and "reflection" $\gamma_{2 i}$ (Fig. 1)

$$
\lambda_{i}=\pi-\psi-\gamma_{2 i} / k_{V i}
$$

where $k_{V i}$ is the recovery factor, which can be estimated similarly to the method proposed by the authors [17] under the condition $k_{V}=\sin \gamma_{2 i} / \sin \gamma_{1 i}$. Taking into account expression (1), the relationship between the characteristic angles is obtained $\gamma_{2 i}$ and $\theta_{i v}$ in the linear approximation in the form

$$
\theta_{i v}\left(\psi, \delta, \gamma_{2 i}\right)=-\left[\pi /\left(2 \alpha_{0}\right)+q_{1}(\psi, \delta)+q_{2}(\psi, \delta) \gamma_{2 i v}\right]
$$

Coefficients $q_{1}(\psi, \delta), q_{2}(\psi, \delta)$ from expression (2) reflect the relationship with the selected parameters $\psi, \delta$ , as the most significant for the studied process of impact mixing of bulk components.

\section{Modeling the distribution of bulk materials according to the characteristic parameters of the impact mixing process}

Considered the motion of rarefied streams of particles "falling" on the bump surface $i=\overline{1, n_{i}}$ from the standpoint of the stochastic approach [32, 33]. The macrosystems of these particles formed at the first stage correspond to a random equilibrium process in the absence of macroscale fluctuations of the states of these macrosystems without energy inflows from outside. Moreover, for the specified process, Markova A.A. (homogeneous, continuous, stationary) stationary solution of the kinetic Fokker-Planck equation is represented in the form

$$
d N_{i v}=C_{i v} \exp \left(-E_{i v} / E_{0 i v}\right) d \Omega_{i v}
$$

where phase volume element $d \Omega_{i v}=d v_{x i v} d v_{y i v}$ has the following form, taking into account the introduced polar coordinate system $\left(r_{i v}, \theta_{i v}\right)$ with the beginning at the center of the Archimedes spiral $r_{A_{i v}}\left(\theta_{i v}\right)$

$$
d \Omega_{i v}=-\omega^{2} r_{i v}^{2} d r_{i v} d \theta_{i v}
$$

The normalization parameter included in expression (3) $C_{i v}$ and energy characteristic $E_{i v}$ are determined similarly to [10] from the system of equations in the form of normalization conditions and the energy balance equation

$$
\int_{\Omega_{i v}} w_{i v} d \Omega_{i v}=1, \quad E_{b i}=E_{r i}
$$

where $E_{b i}$ and $E_{r i}$ are respectively, the energies when the particles of the mixed components interact: first with deformed blades on the belt, then when these particles are removed from the blades when the mixing drum rotates.

Note that the function $w_{i v}\left(\theta_{i v}\right)=N_{i v}^{-1} d N_{i v} / d \theta_{i v}$ from the first equation of system (5) is the result of modeling the distribution of bulk materials by the characteristic parameter $\theta_{i v}$ is the angle of spread of material particles $i=\overline{1, n_{i}}$ blades of each row for the first mixing stage. Application of the obtained dependences (1), (2) for the relationship between the characteristic angles $\lambda_{i}, \gamma_{2 i}$ and $\theta_{i v}$ allows to form the required set of equilibrium differential distribution functions $f_{i v}\left(\gamma_{2 i}\right)=N_{i v}^{-1} d N_{i v} / d \gamma_{2 i}$ in the shape of

$$
\begin{gathered}
f_{i v}\left(\gamma_{2 i}\right)=\sigma_{0 i v} U_{3 i v}\left(\gamma_{2 i}\right) \exp \left(-k_{u} \gamma_{2 i}{ }^{2} / E_{0 i v}\right) \times \\
\times\left(\operatorname{erf}\left\{\sigma_{2 i v}\left[U_{2 i v}\left(\gamma_{2 i}\right)\right]^{2} / U_{3 i v}\left(\gamma_{2 i}\right)\right\}-\right. \\
\left.-\operatorname{erf}\left[\sigma_{2 i v} / U_{3 i v}\left(\gamma_{2 i}\right)\right]\right) \times \\
\times\left\{\exp \left[-10\left(\sigma_{1 i v}+k_{u} \gamma_{2 i}{ }^{2} / E_{0 i v}\right)\right]-\exp \left(-\sigma_{1 i v}\right)\right\}^{-1}
\end{gathered}
$$

where

$$
\begin{aligned}
& U_{1 i v}\left(\theta_{i v}\right) \equiv \varsigma_{0 j}+\varsigma_{1 j} \theta_{i v}, \sigma_{2 i v} \equiv 3\left[s_{1} / E_{0 i v}\right]^{1 / 2}\left(2 \lambda_{0 v}{ }^{2}\right)^{-1}, \\
& U_{2 i v}\left(\theta_{i v}\right) \equiv \lambda_{0 v}+\lambda_{1 v} \theta_{i v}, \sigma_{1 i v} \equiv 3\left[s_{1} / E_{0 i v}\right]^{1 / 2} /\left(2 \lambda_{0 v}{ }^{2}\right),
\end{aligned}
$$




$$
\begin{gathered}
U_{3 i v}\left(\theta_{i v}\right) \equiv\left\{\left[U_{2 i v}\left(\theta_{i v}\right)\right]^{2}+v_{2 j}{ }^{2}\right\}^{1 / 2}, \lambda_{3 v} \equiv \lambda_{0 v} /\left(16 \lambda_{1 v}\right), \\
\sigma_{0 i v} \equiv \\
\lambda_{3 v}\left[\pi k_{u} /\left(E_{0 i v} s_{1} k_{u}^{-1}\right)\right]^{-1 / 2}, s_{1} \equiv k_{u} k_{1 i v} k_{2 i v}, \\
\sigma_{1 i v} \equiv s_{1}\left[E_{0 i v}\left(2{\varsigma_{0 j}}^{2}+\varsigma_{2 j}{ }^{2}\right)\right]^{-1 / 2} .
\end{gathered}
$$

Therefore, when the blades are deformed, for each row $v=\overline{1, k_{0}}$ equilibrium total differential distribution function for the number of particles of bulk material $i=\overline{1, n_{i}}$ by the angle of "reflection" from the bump surface $\gamma_{2 i}$ using expression (6) is given in the following form

$$
F_{i}\left(\gamma_{2 i}\right)=\sum_{v=1}^{k_{0}} f_{i v}\left(\gamma_{2 i}\right)
$$

Note that the coefficients included in expression (6) depend on the sets of parameters of the shock mixing process of granular and/or powder materials: constructive $a_{j_{4}}=\left\{a_{j_{1}}, \psi, H_{1}\right\}$; regime $b_{j_{2}}=\left\{\omega, h_{0}, h_{L}\right\}$, physical and mechanical $c_{j_{3}}=\left\{\rho_{T i}, D_{T i}, k_{u}\right\}$.

\section{Results and Discussion}

Thus, the obtained expressions (6) and (7) make it possible to illustrate the influence of the main parameters of the shock mixing process on a specific example of the formation of flows "reflected" from the baffle surface located at an angle $\psi$ to the movable horizontal conveyor belt. The resulting mixture consist of two components: natural sand GOST 8736-93 $(i=1$, $\left.k_{V}=0.1819, d_{T 1}=2.0 \times 10^{-4} \mathrm{~m}, \rho_{T 1}=1.8 \times 10^{3} \mathrm{~kg} / \mathrm{m}^{3}\right)$ and soda ash GOST 5100-85 $\left(i=2, \quad k_{V}=0.1765\right.$, $\left.d_{T 2}=1.75 \times 10^{-4} \mathrm{~m}, \quad \rho_{T 2}=1.08 \times 10^{3} \mathrm{~kg} / \mathrm{m}^{3}\right), \quad$ value $k_{V}=5.4 \times 10^{-4} \mathrm{~kg} \mathrm{~m} / \mathrm{rad}$.

We set the following sets for the values or ranges of variation of the parameters of the investigated technological operation at the second stage of mixing in a rotary apparatus. The set of design parameters for the impact process of mixing granular media includes: $R_{b}=3.0 \times 10^{-2} \mathrm{~m}, L_{b}=4.5 \times 10^{-2} \mathrm{~m}, h_{0}=3.0 \times 10^{-2} \mathrm{~m}$, $p_{0}=8, k_{0}=3, \psi=0.6109 \mathrm{rad}, H_{1}=2.7 \times 10^{-1} \mathrm{~m}$. The corresponding values of the operating parameters and/or the limits of their change be equal: $h_{L}=0.16 \times 10^{-2} \mathrm{~m}$, $h_{0}=3.0 \times 10^{-2} \mathrm{~m}, \omega=(41.5-52.5) \mathrm{c}^{-1}$.

Obtained graphs for surfaces of functions $f_{i v}\left(\gamma_{2 i}\right), F_{i}\left(\gamma_{2 i}\right)$ shown on Fig. 2, 3. Performed modeling for the equilibrium full differential distribution function $F_{i}\left(\gamma_{2 i}\right)$ for the number of particles of bulk material $i=\overline{1, n_{i}}$ by the angle of "reflection" from the bump surface $\gamma_{2 i}$ according to (7) and its components (6) made it possible to analyze the influence of the selected significant parameters of the process under study: the angular speed of rotation of the drum, the characteristic of the degree of deformation of the blades in the gap between the drum and the conveyor belt $\delta=h_{0} / L_{b}$, the angle of inclination of the bump surface.

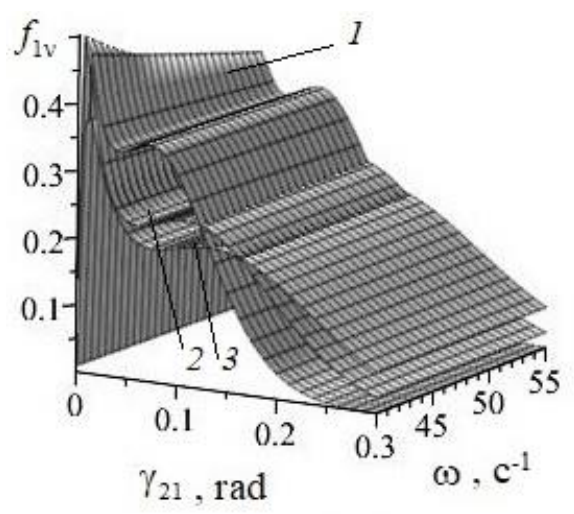

a)

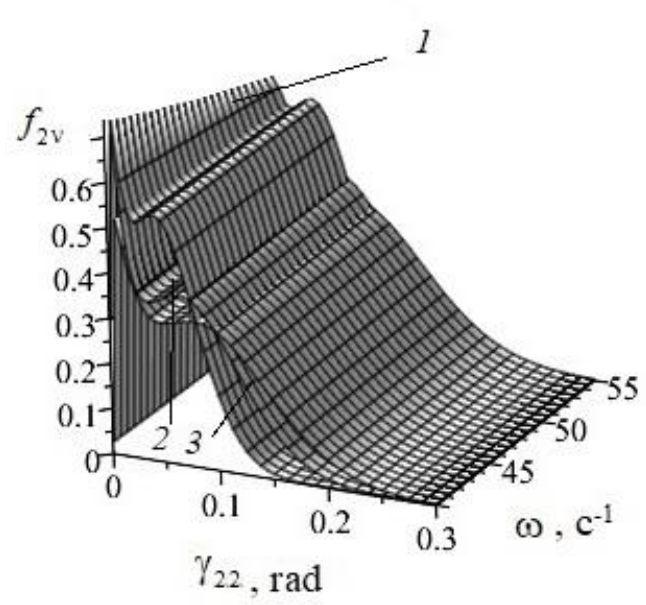

b)

Fig. 2. Dependence $f_{i v}\left(\gamma_{2 i}, \omega\right):$ a) natural sand GOST 8736-93 $(i=1)$; b) soda ash GOST 5100-85 $(i=2) ; \delta=0.67 ; k_{0}=3$; $1-v=1 ; 2-v=2 ; 3-v=3$.

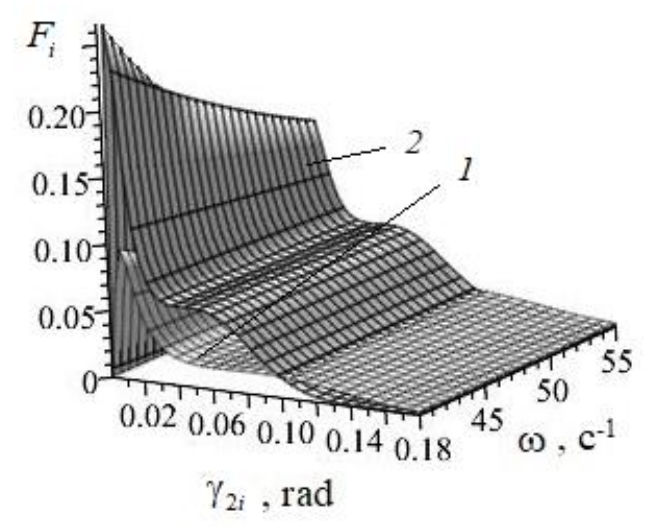

Fig. 3. Dependence $F_{i}\left(\gamma_{2 i}, \omega\right): 1$ - natural sand GOST 8736$93(i=1) ; 2$ - soda ash GOST 5100-85 $(i=2) ; \delta=0.67$; $k_{0}=3 ; \psi=0.6109 \mathrm{rad}$.

According to Fig. 2 on function graphs $f_{i v}\left(\gamma_{2 i}\right)$ for each loose component $(i=1,2)$ there are two characteristic bursts (graphs 1-3, Fig. 2a; graphs 1-3, Fig. 
$2 \mathrm{~b})$. This behavior of the mixed materials is explained by the fact that the formation of rarefied flows "falling" on the inclined surface occurs at stage $1[10]$ and depends on the behavior of the deformed blades of the rotating drum. Most of the mixed components captured by the deformed blades are removed at a small drum rotation angle. The particles remaining on the blades are removed with a further increase in this angle to values not exceeding $0.7 \mathrm{rad}$. When implementing impact mixing, analysis of the graphs of functions $F_{i}\left(\gamma_{2 i}\right), i=1,2$ on

Fig. 3 (graph 1, 2, Fig. 3) shows the retention of the nature of the behavior of particles in "reflected" rarefied flows after interaction with the baffle surface.

On the example of the tested working components, the mechanism of the behavior of their particles during impact mixing is considered. In particular, within the considered limits of variation of the process parameters, the achievement of the first extrema at the angles of "reflection" from the bump surface is observed at values less than $0.02 \mathrm{rad}$ (graph 1, 2, Fig. 3). The result obtained confirms the possibility of reflection of rarefied flows of the mixed components from the baffle surface at comparable angles of "reflection" when the condition for their effective mixing is provided.

The main significant parameters of impact mixing, related to various stages of the process under study, and their effective limits of variation have been established: a) at stage 1 the angular speed of rotation of the drum $\omega$ $=(42,0-54.0) \mathrm{c}^{-1}$ and the degree of deformation of the blades in the gap between the drum and the conveyor belt $\delta=(0.56-0.68) ; b)$ at stage 2 the angle of inclination of the bump surface $\psi=(0.59-062) \mathrm{rad}$.

\section{Conclusions}

In this work, stochastic modeling of impact mixing of bulk components at the second stage of operation of the rotary apparatus is carried out. This device is designed to obtain free-flowing mixtures, including from mineral fertilizers, multifunctional pyrethroid drugs.

In the presented model for the process of impact mixing of bulk components, the following are taken into account:

- many parameters of constructive $a_{j_{4}}=\left\{a_{j_{1}}, \psi, H_{1}\right\}$ and regime $b_{j_{2}}=\left\{\omega, h_{0}, h_{L}\right\}$ types;

- physical and mechanical properties of working materials $c_{j_{3}}=\left\{\rho_{T i}, D_{T i}, k_{u}\right\}$;

- kinematic characteristics $k_{V i}, i=1,2$ their impact interaction with the bump surface.

Reflections of the relationship between the simulation results and the developing theoretical foundations for the design of a rotary mixer for granular and/or powder components are:

- the obtained effective limits of variation of the shock mixing parameters from the sets of constructive $a_{j_{4}}=\left\{a_{j_{1}}, \psi, H_{1}\right\}$ and regime $b_{j_{2}}=\left\{\omega, h_{0}, h_{L}\right\}$ types;
- possible assessment of the quality of the mixture, for example, according to the criterion in the form of the coefficient of heterogeneity [16].

The main conclusions and results of the work include the following.

- A relationship has been established between the characteristics of two stages of mixing bulk materials performed in a rotary apparatus on a conveyor belt (angles of scattering of particles of bulk components during the formation of rarefied flows at stage 1 and angles of their "reflection" from the bump surface at stage 2).

- Within the framework of the energy method Klimontovich Yu.L. [32, 33] proposed a method for constructing complete differential distribution functions for the number of particles of mixed granular components by the angle of "reflection" from the bump surface.

- A stochastic estimate of the relationship of the sought distribution functions with the characteristic parameters of the shock mixing process is given, which have the most significant effect on its efficiency on the example of the tested working components. In this case, the achievement of the first extrema at the angles of "reflection" from the bump surface is performed at values less than $0.02 \mathrm{rad}$.

- The practical application of the simulation results in further studies may be associated with the estimated calculations of the coefficient of heterogeneity of the resulting bulk mixture, as a criterion for the quality of the mixture.

\section{References}

1. Y. Rouphael, G. Colla, Editorial: Biostimulants in Agriculture, Frontiers in Plant Science, 11, 40, 1$7 \quad(4 \quad$ February 2020) DOI: 10.3389/fpls.2020.00040

2. S. Nardi, D. Pizzeghello, M. Schiavon, Ertani, A. Sci. Agric., 73, 18-23 (2016) DOI: 10.1590/01039016-2015-0006

3. S.M. Ensley, Pyrethrins and Pyrethroids, In book: Veterinary Toxicology, 515-520 (March 2018) DOI: 10.1016/B978-0-12-811410-0.000398

4. P. Prashar, S. Shah, Impact of Fertilizers and Pesticides on Soil Microflora in Agriculture, In: E. Lichtfouse (eds.), Sustainable Agriculture Reviews. Sustainable Agriculture Reviews [Springer], 19, Springer, Cham. (2016) DOI: 10.1007/978-3-319-26777-7_8

5. U. Riaz, M. Qazi, Integrated and Site-Specific Fertilizer Application Role in Rice-Wheat Cropping System, Polish Journal of Environmental Studies, 30 (2), 1-8 (2020) DOI: $10.15244 /$ pjoes $/ 125850$

6. Md.W. Aktar, D. Sengupta, A. Chowdhury, Interdisciplinary Toxicology, 2 (1), 1-12 (2009) DOI: $10.2478 / \mathrm{v} 10102-009-0001-7$ 
7. B. Bogomolov, V. Boldyrev, A. Zubarev, V. Meshalkin, V. Men'shikov, Theoretical Foundations of Chemical Engineering, 53 (5), 709-718 (2019) DOI: $10.1134 / \mathrm{S} 0040579519050270$

8. V.V. Kafarov, I.N. Dorokhov, S.Yu. Arutyunov, Sistemnyy analiz khimicheskikh protsessov tekhnolo-gii, Protsessy izmel'cheniya $i$ smeshivaniya sypuchikh materialov [System analysis of chemical technology processes, The processes of grinding and mixing bulk materials] (Moscow, Nauka, 1985, 440)

9. A.E. Lebedev, A.A. Vatagin, M.E. Borisovsky, M.N. Romanova, N.V. Badaeva, I.S. Sheronina, Unit for mixing and compaction of bulk materials (Pat. RF 2624698, IPC B01F3 / 18, bull. no. 19, 07.05.2017)

10. A. Kapranova, D. Bahaeva, D. Stenko, A. Vatagin, A. Lebedev, D. Lichak, E3s Web of Conference, 220, 01060 (2020) DOI: $10.1051 / \mathrm{e} 3$ sconf $/ 202022001060$

11. A.B. Kapranova, I.I. Verloka, D.D. Bahaeva, M.Yu. Tarshis, S.N. Cherpitsky, Frontiers in Energy Research: Process and Energy Systems, 8, 135, 1-11 (7 August 2020) DOI: 10.3389/fenrg.2020.00135

12. A.B. Kapranova, I.I. Verloka, D.D. Bahaeva, About Preparation of the Analytical Platform for Creation of a Cyber-Physical System of Industrial Mixture of Loose Components, In: A. Kravets, A. Bolshakov, M. Shcherbakov (eds) Cyber-Physical Systems: Advances in Design \& Modelling, Studies in Systems, Decision and Control, 259, Springer, Cham, 81-91 (2020) DOI: 10.1007/978-3-030-32579-4_7

13. A.B. Kapranova, I.I. Verloka, Theor. Found. of Chem. Eng., 52 (6), 1004-1018 (2018)

14. A.B. Kapranova, M.N. Bakin, I.I. Verloka, Chem. and Petrol. Eng., 54 (5-6), 287-297 (2018) More about changing the spelling of the author's name Suppl., 54 (7-8), 618 (2018)

15. A. Kapranova, I. Verloka, D. Bahaeva, Factors of Increasing the Performance of the Bulk Material Mixer of the Gravity Type, In: B. Anatolijs, V. Nikolai, S. Vitalii (eds) Proceedings of EECE 2019. EECE 2019, Lecture Notes in Civil Engineering, 70, Springer, Cham, 337-347 (2020) DOI: 10.1007/978-3-030-42351-3_30

16. A.B. Kapranova, I.I. Verloka, P.A. Yakovlev, D.D. Bahaeva, Russian Journal of General Chemistry, 90 (6), 1178-1179 (2020)

17. I. Verloka, A. Kapranova, M. Tarshis, S. Cherpitsky, Int. J. Mech. Eng. Technol., 9 (2), 438-444 (2018)

18. A.B. Kapranova, Theor. Found. of Chem. Eng., 53 (2), 292-304 (2019) DOI: $10.1134 / \mathrm{S} 004057951901007 \mathrm{X}$
19. M. Sen, D. Barrasso, R. Singh, R.A. Ramachandran, Multi-Scale Hybrid CFD-DEMPBM Description of a Fluid-Bed Granulation Process. Processes, 2 (1), 89-111 (2014) DOI: 10.3390/pr2010089

20. T. Geng, L.L. Sau, Ya. Xiaochuan, S.H. Moo, A Dimensionless Analysis of Residence Time Distributions for Continuous Powder Mixing, Powder Technology, 315, 332-338 (2017) DOI: 10.1016/j.powtec.2017.04.007

21. N. Johnson, M. Kendall, A. Stuart, Journal of the Royal Statistical Society Series D (The Statistician), 12 (2), 138 (1962)

22. M.G. Kendall, A. Stuart, Statistical Inference and Statistical Relationship, 4th ed. (Hafner Press. 1986)

23. A. Ghaderi, Continuous mixing of particulate materials, Proceedings of the 4th International Conference for Conveying and Handling of particulate solids, At Budapest, Hungary, 2 (January 2003) DOI: 10.13140/2.1.3487.6801

24. A.T. Bharucha-Reid, Elements of the Theory of Markov Processes and Their Applications (New York: McGraw-Hill, 1960)

25. B.I. Leonchik, V.P. Mayakin, Izmereniya $v$ rasseyannykh potokakh [Measurements in dispersed flows] (Moscow: Energy, 1985, 248)

26. A. Brownian Papoulis, Movement and Markoff Processes. Ch. 15 in Probability, Random Variables, and Stochastic Processes, 2nd ed. (New York: McGraw-Hill, 1984, 515-553)

27. G. Röpke, Statistical Mechanics for NonEquilibrium (Berlin, German Publishing House of Sciences, 1987)

28. Y. Zhuang, X. Chen, D. Liu, Chemical Engineering Journal, 291, 206-214 (2016)

29. F.G. Akhmadiev, I.T. Nazipov, Theoretical Foundations of Chemical Engineering, 47 (2), 136 (2013)

30. H.G. Dehling, T. Gottschalk, A.C. Hoffmann, Stochastic Modeling in Process Technology (Elsevier Science, London, 2007, 279)

31. V. Mizonov, I. Balagurov, H. Berthiaux, C. Gatumel, Particuology, 31, 80-86 (2016)

32. Yu.L. Klimontovich, Turbulent Motion and the Structure of Chaos, Series: Fundamental Theories of Physics, Springer Netherlands, 42, 401 (1991) DOI: 10.1007/978-94-011-3426-2

33. Yu.L. Klimontovich, Turbulentnoe dvizhenie $i$ struktura khaosa: Novyy podkhod $k$ statisticheskoy teorii otkrytykh system [Turbulent motion and chaos structure: A new approach to the statistical theory of open systems], 4rd ed. (Moscow, LENAND, 2014, 328)

34. A.B. Kapranova, A.E. Lebedev, A.M. Meltser, S.V. Neklyudov, E3s Web of Conference, 140, 06005 (2019) DOI: $10.1051 /$ e3sconf/202022001060 
35. A.B. Kapranova, A.E. Lebedev, S.V. Neklyudov, A.M. Melzer, Frontiers in Energy Research: Process and Energy Systems, 8, 32, 1-17 (March 2020) DOI: $10.3389 /$ fenrg.2020.00032

36. A.B. Kapranova, A.E. Lebedev, A.M. Meltser, S.V. Neklyudov, E3s Web of Conference, 220, 01073, (2020) DOI: 10.1051/e3sconf/202022001073

37. A.I. Zaitsev, D.O. Bytev, Udarnyye protsessy v dispersno-plenochnykh siste-makh [Impact processes in the dispersion-film systems] (Moscow: Chemistry, 1994, 176). 